\title{
Scattering from metamaterial coated nihility sphere
}

\author{
A. Ghaffar ${ }^{1 *}$, M. M. Hussan ${ }^{1}$, Majeed A. S. Alkanhal ${ }^{2}$ and Sajjad ur Rehman²
}

\begin{abstract}
Background: To show the Back-Scattering efficiencies of nihility sphere become non-zero, scattering from metamaterial coated nihility sphere has been carried out in the presented work.

Methods: The field phasors are expanded in terms of spherical wave vector function along with assumed scattering coefficients. Boundaries conditions are applied at each interface, i.e., free space- metamaterial coating and metamaterial coating-nihility sphere core.

Results: Scattering coefficients are obtained by using boundary conditions at each interface. The scattering efficiencies are obtained in graphical form by varying both permittivity and permeability of metamaterial using MATHEMATICA software. Under some special conditions obtained results are compared with already published literature to show the correctness of present formulations.

Conclusion: It is observed that Back-Scattering efficiencies of nihility sphere becomes non-zero when we introduce a metamaterial coating layer on it. It is also noticed that the scattering efficiencies are dependent on coating thickness as well as permittivity and permeability of metamaterials.
\end{abstract}

Keywords: Nihility, Sphere, Metamaterial, Scattering, Forward-scattering efficiency, Back-scattering and Extinction efficiency

\section{Background}

A lot of engineers, researchers and professional's belonging to optical society pay a vital consideration towards those materials which were artificially designed, which we often call as metamaterial. Because metamaterials are tremendously good to tune and control the electromagnetic properties at vide range of frequencies which is not possible for naturally occurring materials. Many researchers used metamaterials as filters, phase shifters, perfect reflectors, electromagnetic invisibility cloak, and wave guiders [1-5]. Some characteristic metamaterials which were being considered widely in already published work are plasma, Perfect Electromagnetic Conductor(PEMC), chiral [6], chiral nihility and split ring resonator (SRR) [7-9]. The nihility medium was considered as "the electromagnetically nilpotent" and has the most surprising impression in the field of electromagnetics and optics, in this material the relative permeability and relative permittivity both have zero magnitude.

\footnotetext{
* Correspondence: aghaffar16@uaf.edu.pk

${ }^{1}$ Department of Physics, University of Agriculture, Faisalabad, Pakistan

Full list of author information is available at the end of the article
}

Nihility material introduced by Lakhtakia $[10,11]$ attracted many researchers because wave cannot propagate in nihility medium. Lakhtakia postulated that nihility medium is a medium whose permittivity and permeability both are null valued [10-13]. Due to null valued permittivity and permeability this medium does not allow the wave to propagate. Maxwell equation under nihility conditions reduced into below form

$$
\begin{aligned}
& \nabla \times \boldsymbol{E}=0 \\
& \nabla \times \boldsymbol{H}=0
\end{aligned}
$$

Many researchers used nihility material and performed many experiments regarding nihility waveguides, electromagnetic/plane wave scattering from different objects made up of nihility material [11, 12, 14-18]. Lakhtakia derived the mathematical solutions for scattering of electromagnetic wave from sphere made up of nihility and concluded that its Back-Scattering efficiency is exactly zero, he also reported that both Forward-scattering and Extinction efficiencies of nihility sphere showed larger magnitude than perfect electric and perfect magnetic conductors [12]. In 
addition Lakhtakia and Geddes proposed the analytical solution of plane wave scattering from infinite long nihility cylinder [11]. Ahmad et al., analytically solved the plane wave scattering by considering infinite long nihility cylinder coated with metamaterials and deduced that metamaterial layer can tune the scattering efficiencies [19]. The scattering characteristics of plane wave scattering from infinite cylinder made up of nihility covered with chiral layer also has been studied by Ahmed and Naqvi [20]. Sobia et al., analytically formulated the scattering of the electromagnetic wave from chiral coated nihility cylinder embedded in chiral media [21]. Yaqoob et al., used anisotropic plasma as a coating material and solved the scattering problem from nihility cylinder placed in chiral medium, and deduced that chiral and anisotropic plasma parameters can be used to tune the scattering efficiencies [22].

In the present paper, the problem of electromagnetic wave scattering from nihility sphere coated with metamaterial, i.e., Double Positive (DPS), Double Negative (DNG), Epsilon Negative (EMG) and Mu negative (MNG) are formulated mathematically and computationally. For the simplicity of mathematical formulations coating layer has been considered of uniform thickness. In order to expand the incident, the scattered and the transmitted electromagnetic field, spherical wave vector function are used. For mathematical solutions, the proposed geometry of subjected problem was sliced into three regions free space as region 0 , metamaterial coating as region 1 and Nihility sphere as region 2, shown in the Fig. 1. By applying the boundaries conditions at each interface a set of eight equations are obtained which are used to find the scattering coefficients. These obtained scattering coefficients are used to calculate the scattering efficiencies, which were then

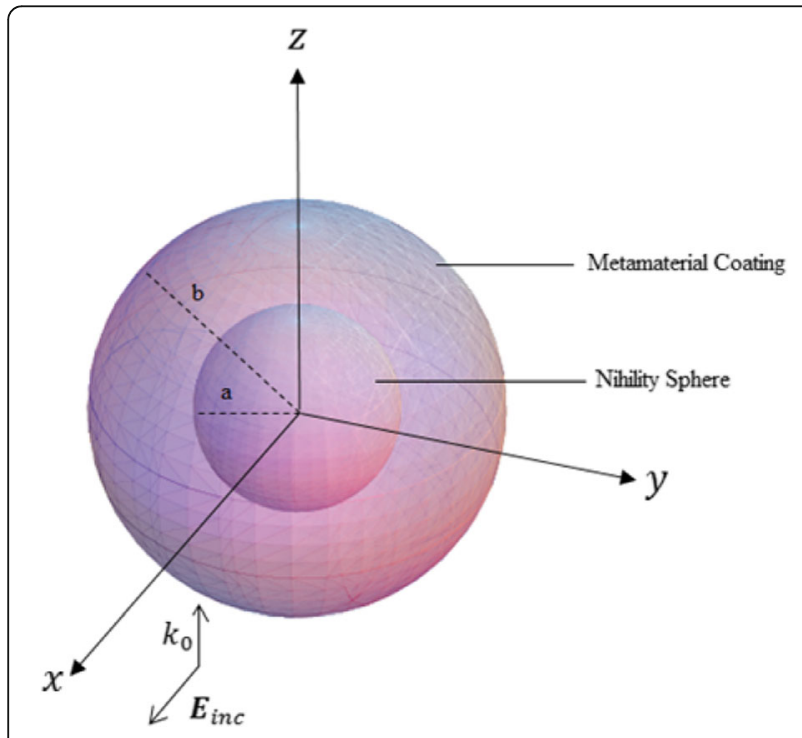

Fig. 1 Metamaterial coated nihility sphere compared with already published literature to show the accuracy of present formulation. In this paper the time dependence $e^{-j \omega t}$ was considered throughout the mathematical formulation.

\section{Analytical Formulations and Method}

The propose geometry of the presented problem of plane wave scattering from nihility sphere coated with DPS, DNG, MNG or ENG material is depicted in Fig. 1. Where $b$ and $a$ represents the radius of sphere with coating and without coating respectively. The outer medium i.e. $\rho \geq b$ is free space having wave number $k_{0}=\omega \sqrt{\epsilon_{0} \mu_{0}}$ is represented by region 0 . The coating medium $a<\rho<b$ with wave number $k_{1}=\omega \sqrt{\epsilon_{1} \mu_{1}}$ is termed as region 1 . Region $\rho<a$ with wavenumber $k_{2}=\omega \sqrt{\epsilon_{2} \mu_{2}}$ is represented by region 2.

In spherical coordinate system $(r, \theta, \phi)$ the spherical wave vector functions are given as.

$$
\begin{aligned}
& \boldsymbol{M}_{\sigma m n \gamma}^{(l)}=\nabla \times\left[\boldsymbol{r} Y_{\sigma m n}(\theta, \phi) R_{n}^{l}\left(k_{\gamma} r\right)\right] \\
& \boldsymbol{N}_{\sigma m n \gamma}^{(l)}=\frac{1}{k_{\gamma}}\left[\nabla \times \boldsymbol{M}_{\sigma m n \gamma}^{(l)}\right]
\end{aligned}
$$

Where $Y_{\text {бтn }}(\theta, \phi)$ represents spherical harmonic, the most significant property of spherical harmonics is its parity. Here $\sigma$ represents the parity of spherical harmonics, which is even when $\sigma=e$ and odd when $\sigma=o$. The radial function $R_{n}^{l}\left(k_{\gamma} r\right)$ transform to the spherical Bessel $J_{n}\left(k_{\gamma} r\right)$, Spherical Neumann function $n_{n}\left(k_{\gamma} r\right)$ and spherical Hankel function $h_{n}\left(k_{\gamma} r\right)$ corresponding to $l=1,2,3$ respectively. Where subscript $\gamma$ represents the appropriate wave number i.e., $\gamma=0,1,2$ Represents the region 0 (free space) wave number and region 1 wave number $k_{1}$ and so on.

A Plane wave traveling in $+z$ direction with its electric field polarized in the positive $x$ direction is incident on metamaterial coated nihility sphere. The incident electromagnetic field in terms of spherical vector wave functions is given as.

$$
\begin{aligned}
& E_{\mathrm{inc}}(r)=E_{0} \sum_{n=1}^{\infty} i^{n} \frac{(2 n+1)}{n(n+1)}\left(\boldsymbol{M}_{\mathrm{oln}}^{(1)}-i \boldsymbol{N}_{\mathrm{eln}}^{(1)}\right) \\
& H_{\mathrm{inc}}(r)=-\frac{k_{0}}{\omega \mu_{0}} E_{0} \sum_{n=1}^{\infty} i^{n} \frac{(2 n+1)}{n(n+1)}\left(\boldsymbol{M}_{\mathrm{eln}}^{(1)}+i \boldsymbol{N}_{\mathrm{oln}}^{(1)}\right)
\end{aligned}
$$

Where $k_{0}$ and $\mu_{0}$ is the wavenumber and permeability of free space.

The scattered field can be written as

$$
E_{\mathrm{sc}}(r)=E_{0} \sum_{n=1}^{\infty} i^{n} \frac{(2 n+1)}{n(n+1)}\left(i a_{n} \boldsymbol{N}_{\mathrm{eln}}^{(3)}-b_{n} \boldsymbol{M}_{\mathrm{oln}}^{(3)}\right)
$$




$$
H_{\mathrm{sc}}(r)=\frac{k_{0}}{\omega \mu_{0}} E_{0} \sum_{n=1}^{\infty} i^{n} \frac{(2 n+1)}{n(n+1)}\left(i b_{n} \boldsymbol{N}_{\mathrm{oln}}^{(3)}+a_{n} \boldsymbol{M}_{\mathrm{eln}}^{(3)}\right)
$$

Electromagnetic field that transmitted in region 1 can be represented as

$$
\begin{gathered}
E_{I}(r)=E_{0} \sum_{n=1}^{\infty} i^{n} \frac{(2 n+1)}{n(n+1)}\left(c_{n} \boldsymbol{M}_{\mathrm{oln}}^{(1)}+d_{n} \boldsymbol{M}_{\mathrm{oln}}^{(2)}-i e_{n} \boldsymbol{N}_{\mathrm{eln}}^{(1)}\right. \\
\left.-i f_{n} \boldsymbol{N}_{\mathrm{eln}}^{(2)}\right) \\
H_{I}(r)=\frac{-\mathrm{k}_{1}}{\omega \mu_{1}} E_{0} \sum_{n=1}^{\infty} i^{n} \frac{(2 n+1)}{n(n+1)}\left(e_{n} \boldsymbol{M}_{\mathrm{eln}}^{(1)}+f_{n} \boldsymbol{M}_{\mathrm{eln}}^{(2)}-i c_{n} \boldsymbol{N}_{\mathrm{oln}}^{(1)}\right. \\
\left.-i d_{n} \boldsymbol{N}_{\mathrm{oln}}^{(2)}\right)
\end{gathered}
$$

Here $\mathrm{k}_{1}$ represents the wavenumber in region 1 having $\mu_{1}$ its permeability. Electromagnetic Field present in region 2 is

$$
\begin{aligned}
& E_{I I}(r)=E_{0} \sum_{n=1}^{\infty} i^{n} \frac{(2 n+1)}{n(n+1)}\left[g_{n} \boldsymbol{M}_{\mathrm{oln}}^{(1)}-i h_{n} \boldsymbol{N}_{\mathrm{eln}}^{(1)}\right] \\
& H_{I I}(r)=\frac{-\mathrm{k}_{2}}{\omega \mu_{2}} E_{0} \sum_{n=1}^{\infty} i^{n} \frac{(2 n+1)}{n(n+1)}\left[l_{n} \boldsymbol{M}_{\mathrm{eln}}^{(1)}+i l_{n} \boldsymbol{N}_{\mathrm{oln}}^{(1)}\right]
\end{aligned}
$$

The analytically solved boundary conditions at both interface are listed below

$$
\left.\begin{array}{ll}
E_{\theta}^{i n c}+E_{\theta}^{s c a t}=E_{\theta}^{I} & \rho=b \\
H_{\theta}^{i n c}+H_{\theta}^{s c a t}=H_{\theta}^{I} & \rho=b \\
E_{\theta}^{I}=E_{\theta}^{I I} & \rho=a \\
H_{\theta}^{I}=H_{\theta^{I I}} & \rho=a
\end{array}\right\}
$$

By using field Eqs. 5, 6, 7, 8, 9, 10, 11 and 12 in the Eq. 13 set of eight equations are obtained in terms of scattering coefficients.

$$
\begin{aligned}
& h_{n}\left(r_{0}\right) b_{n}+j_{n}\left(r_{1}\right) c_{n}+n_{n}\left(r_{1}\right) d_{n}=j_{n}\left(r_{0}\right) \\
& r_{1}\left[r_{0} h_{n}\left(r_{0}\right)\right]^{\prime} a_{n}+r_{0}\left[r_{1} j_{n}\left(r_{1}\right)\right]^{\prime} e_{n}+r_{0}\left[r_{1} n_{n}\left(r_{1}\right)\right]^{\prime} f_{n} \\
& \quad=r_{1}\left[r_{0} j_{n}\left(r_{0}\right)\right]^{\prime}
\end{aligned}
$$

$$
\begin{aligned}
& \eta_{0}^{-1} h_{n}\left(r_{0}\right) a_{n}+\eta_{1^{-1}} j_{n}\left(r_{1}\right) e_{n}+\eta_{1^{-1}} n_{n}\left(r_{1}\right) f_{n} \\
& \quad=\eta_{0^{-1}} j_{n}\left(r_{0}\right)
\end{aligned}
$$

$$
\begin{aligned}
& \eta_{0}^{-1} r_{1}\left[r_{0} h_{n}\left(r_{0}\right)\right]^{\prime} b_{n}-\eta_{1^{-1}} r_{0}\left[r_{1} j_{n}\left(r_{1}\right)\right]^{\prime} c_{n}-\eta_{1^{-1}} r_{0}\left[r_{1} n_{n}\left(r_{1}\right)\right]^{\prime} d_{n} \\
& =\eta_{0^{-1}} r_{1}\left[r_{0} j_{n}\left(r_{0}\right)\right]^{\prime} \\
& j_{n}\left(r_{2}\right) c_{n}+n_{n}\left(r_{2}\right) d_{n}-j_{n}\left(r_{3}\right) g_{n}=0 \\
& r_{3}\left[r_{2} j_{n}\left(r_{2}\right)\right]^{\prime} e_{n}+r_{3}\left[r_{2} n_{n}\left(r_{2}\right)\right]^{\prime} f_{n}-r_{2}\left[r_{3} j_{n}\left(r_{3}\right)\right]^{\prime} h_{n}=0 \\
& \eta_{1}^{-1} j_{n}\left(r_{2}\right) e_{n}+\eta_{1^{-1}} n_{n}\left(r_{2}\right) f_{n}-\eta_{2^{-1}} j_{n}\left(r_{3}\right) h_{n}=0 \\
& \eta_{1}^{-1} r_{3}\left[r_{2} j_{n}\left(r_{2}\right)\right]^{\prime} c_{n}+\eta_{1^{-1}} r_{3}\left[r_{2} n_{n}\left(r_{2}\right)\right]^{\prime} d_{n}-\eta_{2^{-1}} r_{2}\left[r_{3} j_{n}\left(r_{3}\right)\right]^{\prime} g_{n}=0
\end{aligned}
$$

Where the impedance is defined as $\eta_{n}=\sqrt{\frac{\mu_{n}}{\epsilon_{n}}}$. These equations are solved for scattering coefficients $a_{n}$ and $b_{n}$ which are then used to solve the scattering efficiency, Forward-scattering efficiency, Back-scattering and Extinction efficiency as given below where $k_{0} b=$ $r_{0}, k_{1} b=r_{1}, k_{1} a=r_{2}, k_{2} a=r_{3}$

The energy flow (Poynting vector) was obtained by implementing the scattered field solution and can be written as [23]

$$
\begin{aligned}
& S_{i}=\frac{1}{2} \operatorname{Re}\left(E_{i} \times H_{i}^{*}\right) \\
& S_{s}=\frac{1}{2} \operatorname{Re}\left(E_{s} \times H_{s}^{*}\right) \\
& S_{\text {ext }}=\frac{1}{2} \operatorname{Re}\left(E_{i} H_{s}^{*}-E_{s} H_{i}^{*}\right)
\end{aligned}
$$

Where $S_{i}$ and $S_{s}$ are the corresponding Poynting vector associated with incident and scattered field respectively, while $S_{\text {ext }}$ represents the Poynting vector induced due to interaction between incident and scattered

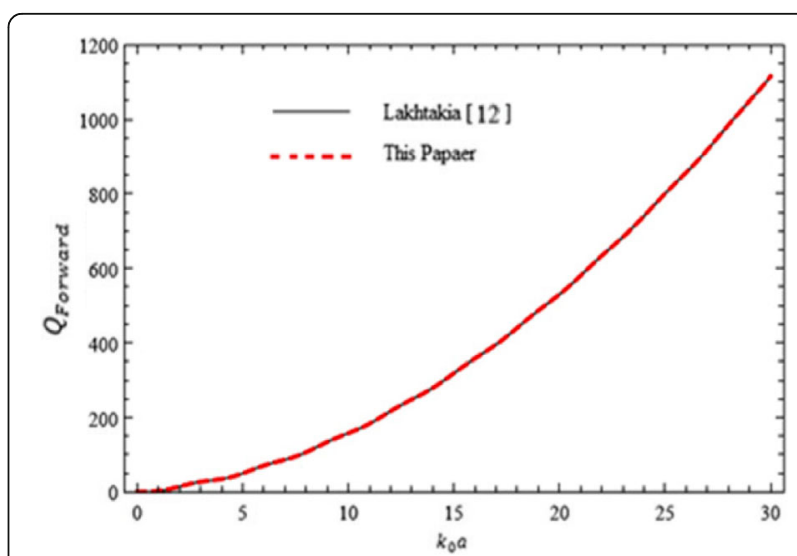

Fig. 2 Forward-scattering efficiency as a function of $k_{0} a$ when coating layer has been removed by setting coating layer parameter equals to core i.e. nihility $b=a, \epsilon_{2}=\epsilon_{1}, \mu_{2}=\mu_{1}$ 


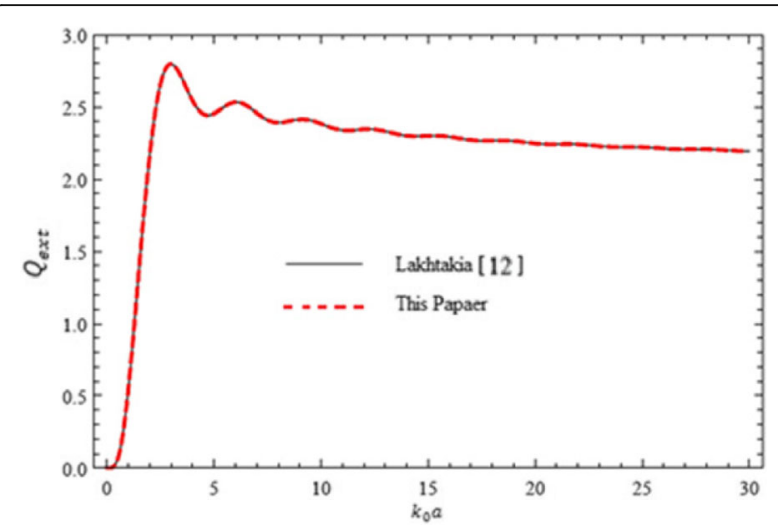

Fig. 3 Extinction efficiency as a function of $k_{0} a$ when coating layer has been removed by setting coating layer parameter equals to core i.e. nihility $b=a, \boldsymbol{\epsilon}_{2}=\boldsymbol{\epsilon}_{1}, \mu_{2}=\mu_{1}$

electromagnetic waves. By following standard Mie theory, if we integrate the above equation over a large sphere, various scattering efficiencies can be obtained [24]. This yields the extinction efficiency and scattering efficiencies (forward scattered and backscattered) [23, 24] as presented in Eqs. 22, 23 and 24.

$$
\begin{aligned}
& Q_{\text {ext }}=\frac{2}{r_{0}^{2}} \sum_{n=1}^{\infty}(2 n+1) \Re\left(a_{n}+b_{n}\right) \\
& Q_{\text {forward }}=\frac{1}{r_{0}^{2}}\left|\sum_{n=\alpha_{\infty}}^{\infty}(2 n+1)\left(a_{n}+b_{n}\right)^{2}\right|^{2} \\
& Q_{\text {backward }}=\frac{1}{r_{0}^{2}}\left|\sum_{n=1}^{n}(-1)^{n}(2 n+1)\left(b_{n}-a_{n}\right)^{2}\right|^{2}
\end{aligned}
$$

By using values of scattering coefficients $a_{n}$ and $b_{n}$ in above equation nihility condition is applied i.e., $\epsilon_{2}=0$ and $\mu_{2}=0$, and results are obtained.

\section{Results and discussions}

In the previous section, mathematical formulations of plane wave scattering from nihility sphere coated with metamaterial have been calculated. In order to gain the more detail of the presented work and also to check the correctness of mathematical formulations Forward-scattering and Extinction efficiencies are plotted under some special conditions. The obtained results are then compared with already published literature [12] which depicted the accuracy of the presented formulation. When we removed the coating layer

Table 1 Material conversion values

\begin{tabular}{lll}
\hline Material & $\epsilon_{1}$ & $\mu_{1}$ \\
\hline Nihility & $\approx 0$ & $\approx 0$ \\
PEC & $\approx \infty$ & $\approx 0$ \\
PMC & $\approx 0$ & $\approx \infty$ \\
\hline
\end{tabular}

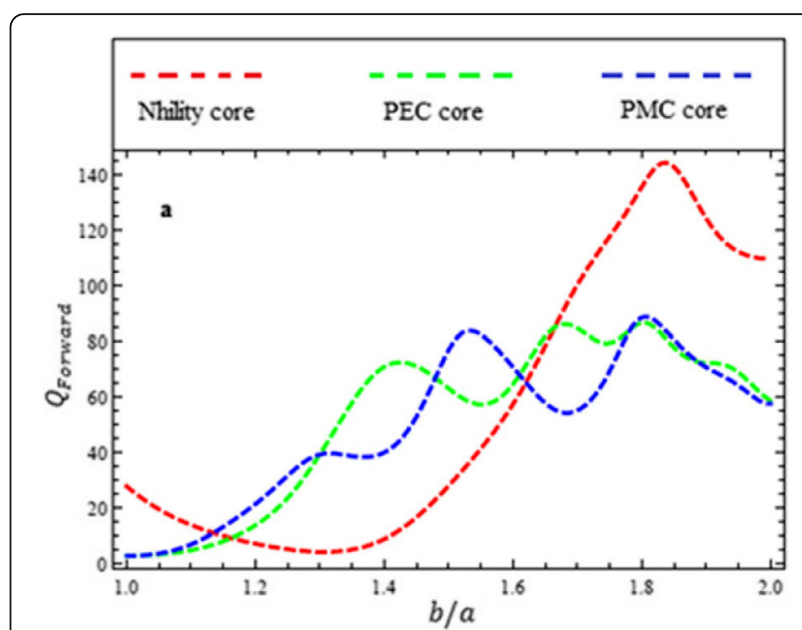

Fig. 4 Forward-scattering efficiency of nihility, PEC and PMC sphere as a function of b/a when $\boldsymbol{\epsilon}_{1}=2.54, \mu_{1}=1, a=0.5 \times \lambda, f=1 \mathrm{GHz}$.

by considering $b=a, \epsilon_{2}=\epsilon_{1}, \mu_{2}=\mu_{1}$ and by applying nihility boundary conditions i.e., $\epsilon_{1} \rightarrow 0, \mu_{1} \rightarrow 0$ the presented problem reduced to electromagnetic wave scattering from nihility sphere. Then by using these specified conditions Forward-scattering and Extinction efficiencies are plotted by using MATHEMATICA software and compared with already published work which are in great agreement as shown in Figs. 2 and 3.

To compare the obtained results of nihility core coated with metamaterial with PEC and PMC sphere coated with metamaterial conversion conditions presented in Table 1 were enforced.

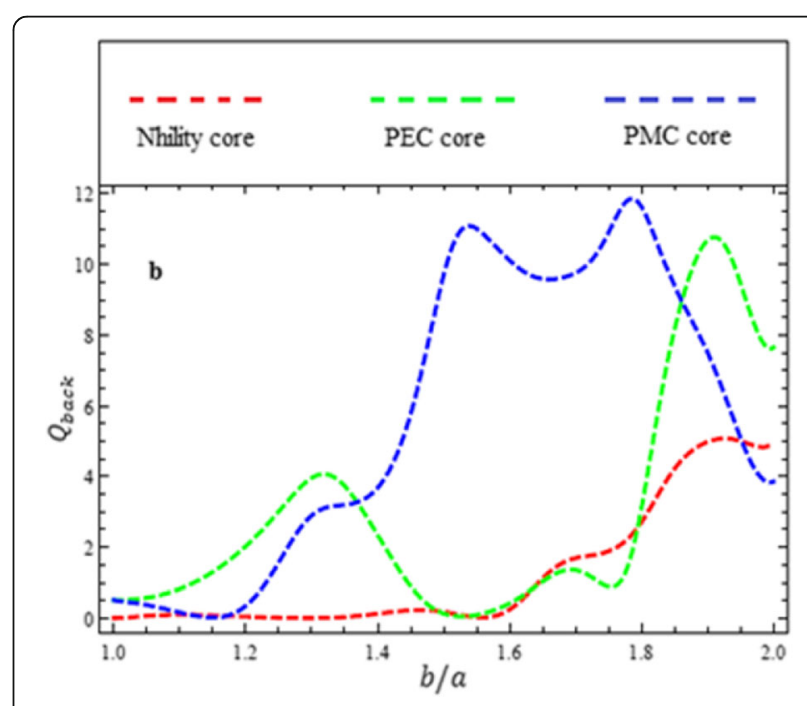

Fig. 5 Back-scattering efficiency of nihility, PEC and PMC sphere as a function of b/a when $\boldsymbol{\epsilon}_{1}=2.54, \mu_{1}=1, a=0.5 \times \lambda, f=1 \mathrm{GHz}$ 


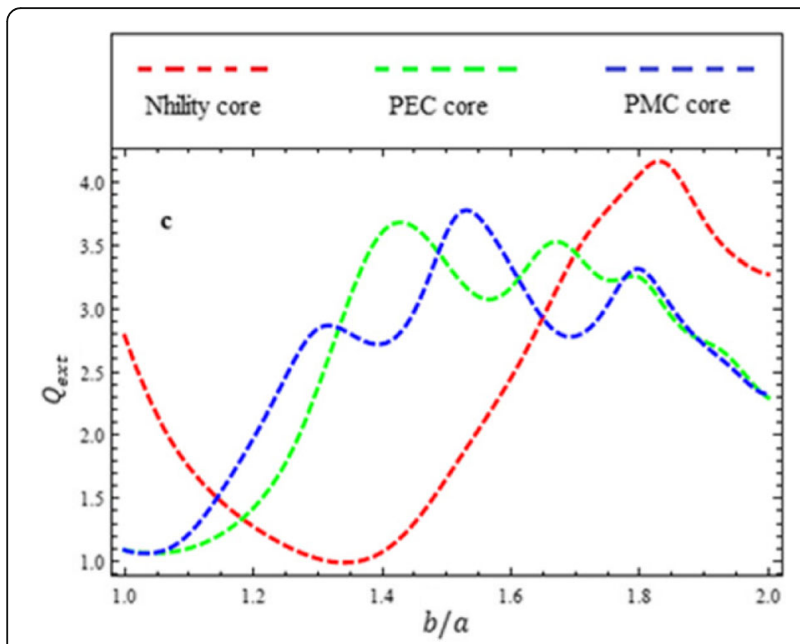

Fig. 6 Extinction efficiency of nihility, PEC and PMC sphere as a function of b/a when $\boldsymbol{\epsilon}_{1}=2.54, \mu_{1}=1, a=0.5 \times \lambda, f=1 \mathrm{GHz}$.

\section{DPS Coating}

Figure 4 represents the comparison between ForwardScattering efficiencies of nihility, PEC and PMC sphere when coated with DPS metamaterial as a function of coating layer thickness with following parameters $\epsilon_{1}=$ 2.54, $\mu_{1}=1, a=0.5 \times \lambda, f=1 \mathrm{GHz}$. By analyzing Fig. 4 we deduced that the Forward-scattering efficiency of nihility sphere is higher than that of PEC sphere and PMC sphere at higher values of coating thickness and lower at lower values of coating thickness. Figure 5 shows the compared results of Back-Scattering efficiencies versus coating layer thickness when nihility, PEC and PMC sphere covered with layer of DPS metamaterial by inserting following parameters $\epsilon_{1}=2.54, \mu_{1}=1, a=0.5 \times \lambda, f=$ $1 \mathrm{GHz}$. Figure 5 reflects that the Back-Scattering efficiency of nihility sphere is lower (but not zero as in uncoated

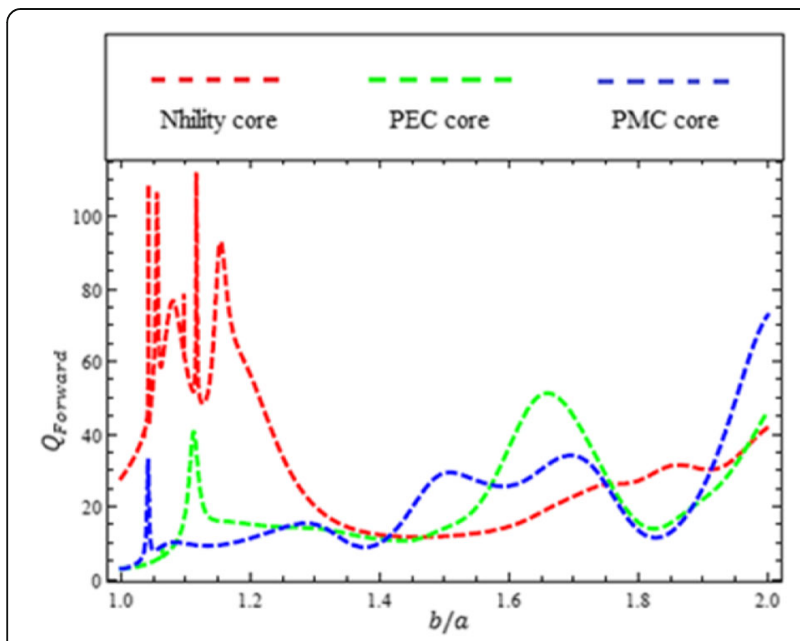

Fig. 7 Forward-scattering efficiency of nihility, PEC and PMC sphere as a function of b/a when $\boldsymbol{\epsilon}_{1}=-2.54, \mu_{1}=-1, a=0.5 \times \lambda, f=1 \mathrm{GHz}$

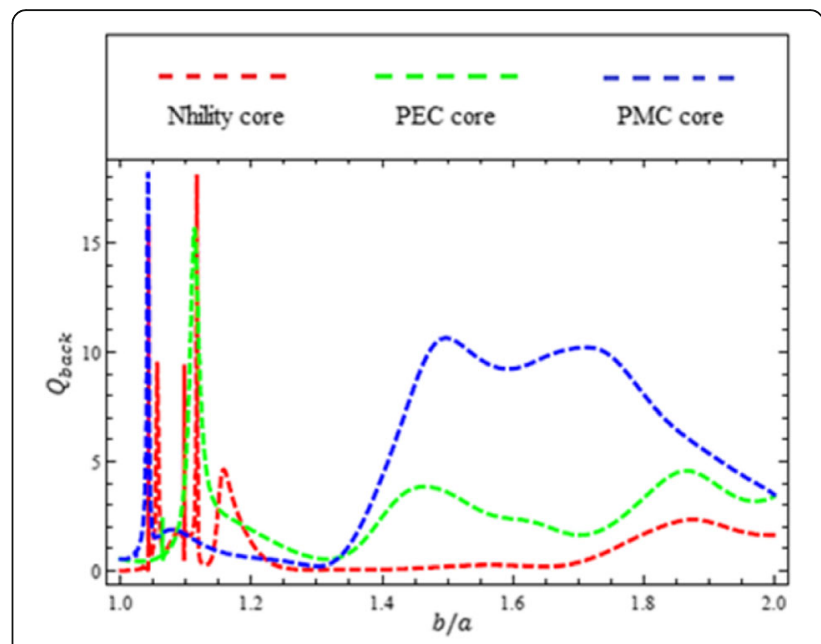

Fig. 8 Back-scattering efficiency of nihility, PEC and PMC sphere as a function of b/a when $\epsilon_{1}=-2.54, \mu_{1}=-1, a=0.5 \times \lambda, f=1 \mathrm{GHz}$

case [12]) than PEC sphere and PMC sphere when coated with DPS metamaterial layer. In Fig. 6 the Extinction efficiencies of different sphere core i.e., nihility, PEC and PMC are coated with DPS metamaterial and are comparatively plotted against the coating thickness by considering the same parameters as that used in Figs. 4 and 5. By analyzing Fig. 6, we reported that the extinction efficiency of nihility sphere is higher as compared to PEC and PMC cases at higher values of coating thickness and vice versa.

\section{DNG Coating}

Forward-scattering, Back-scattering and Extinction efficiencies of nihility sphere are plotted along with PEC and PMC sphere when coated with DNG metamaterial when $\epsilon_{1}=-2.54, \mu_{1}=-1, a=0.5 \times \lambda, f=1 G H z$ as shown

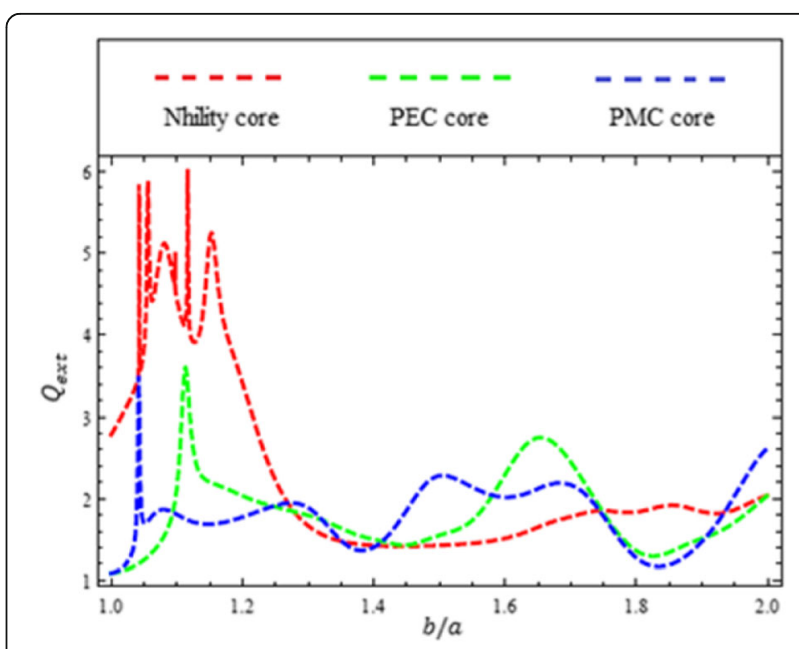

Fig. 9 Extinction efficiency of nihility, PEC and PMC sphere as a function of b/a when $\boldsymbol{\epsilon}_{1}=-2.54, \mu_{1}=-1, a=0.5 \times \lambda, f=1 \mathrm{GHz}$ 


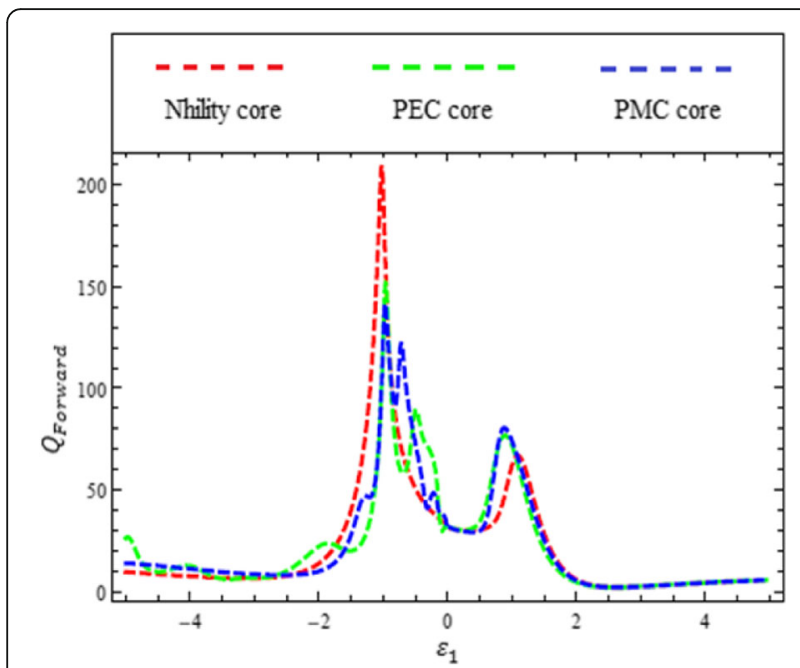

Fig. 10 Comparison between Forward-Scattering efficiencies of nihility, PEC and PMC sphere as a function of $\boldsymbol{\epsilon}_{1}$ when $\mu_{1}=-1$, $a=0.4 \times \lambda, b=1.5 \times a, f=1 \mathrm{GHz}$

in Figs. 7, 8 and 9 respectively. From Figs. 7, 8 and 9 we concluded that when the nihility sphere is coated with DNG metamaterial the behavior of Forward-scattering, Back-scattering and Extinction efficiencies are more sensitive than DPS coated nihility sphere and ForwardScattering, Back-scattering and Extinction efficiencies depict higher magnitude then PEC and PMC at lower values of coating thickness and vice versa.

\section{ENG and MNG Coating}

Forward-scattering, Back-Scattering and Extinction efficiencies of nihility sphere when coated with metamaterial by substituting following parameters: $\mu_{1}=-1, a=0.4 \times \lambda$, $b=1.5 \times a, f=1 \mathrm{GHz}$ were compared with PEC and PMC sphere as shown in Figs. 10, 11 and 12. From Fig. 10 we

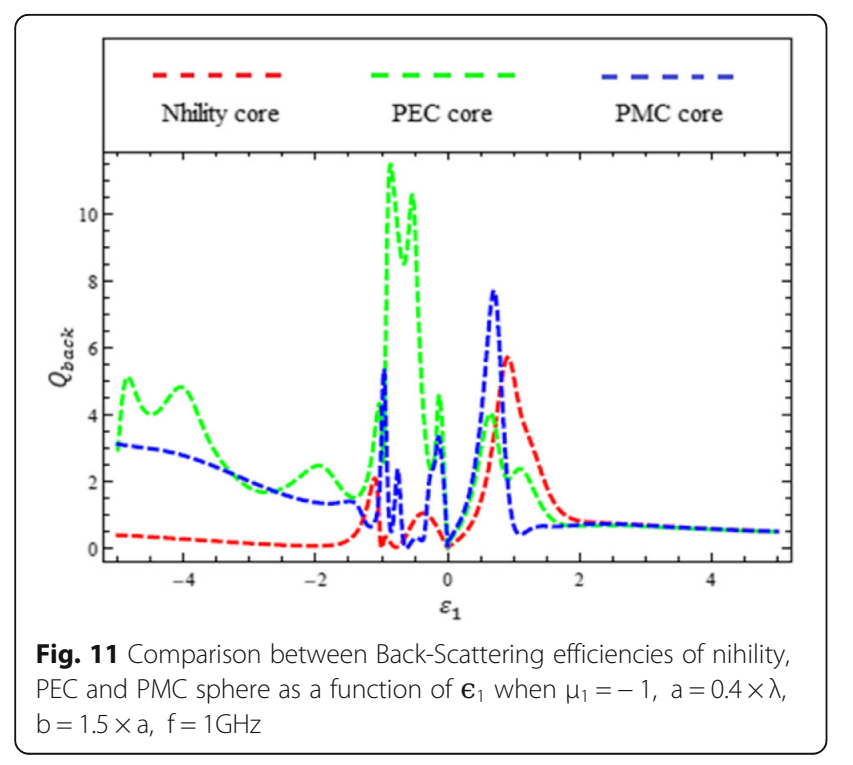

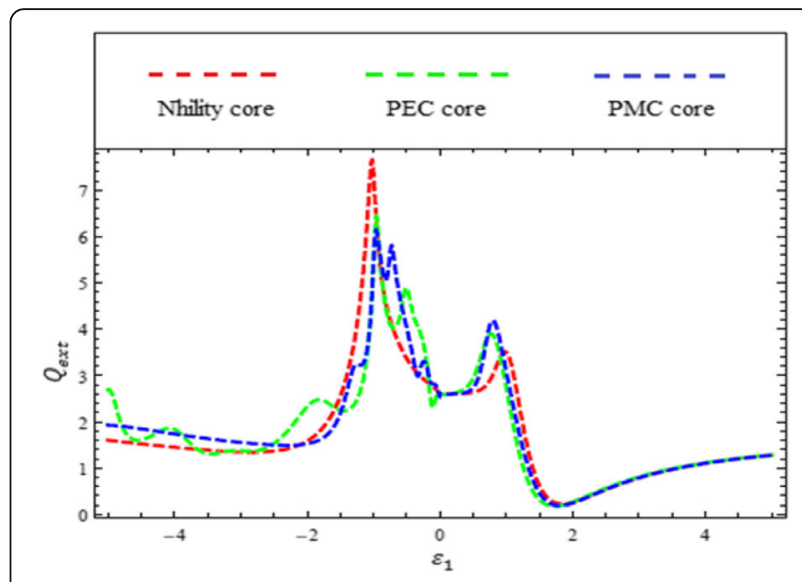

Fig. 12 Comparison between Extinction efficiencies of nihility, PEC and PMC sphere as a function of $\boldsymbol{\epsilon}_{1}$ when $\mu_{1}=-1, a=0.4 \times \lambda$, $b=1.5 \times a, f=1 \mathrm{GHz}$

concluded that the both Forward-scattering and Extinction efficiencies of nihility core depict lower magnitude than PEC and PMC sphere when we considered only MNG $\left(\epsilon_{1}=0\right.$ to 5$)$ coating while, show higher magnitude than PEC and PMC when coating has been considered of DNG $\left(\epsilon_{1}-2\right.$ to -1$)$. It was further deduced that the Back-scattering efficiency of nihility is lower than PEC and PMC, which can be confirmed from Fig. 11.

Forward-scattering, Back-Scattering and Extinction efficiencies of nihility sphere as a function of $\mu_{1}$ when coated with metamaterial by considering following parameters $\epsilon_{1}=-2.54, a=0.4 \times \lambda, b=1.5 \times a, f=1 \mathrm{GHz}$ are compared with PEC and PMC sphere as shown in Figs. 13, 14 and 15 respectively. By analyzing Figs. 13 and 15 it is reported that the magnitude of both Forward-Scattering and Extinction efficiencies corresponding to nihility sphere depicts higher magnitude than PEC

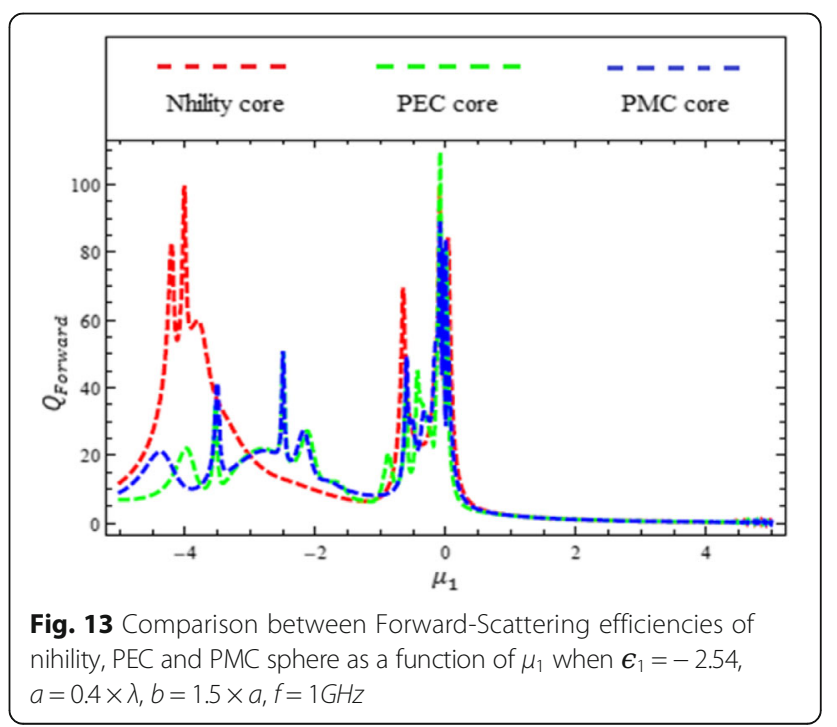




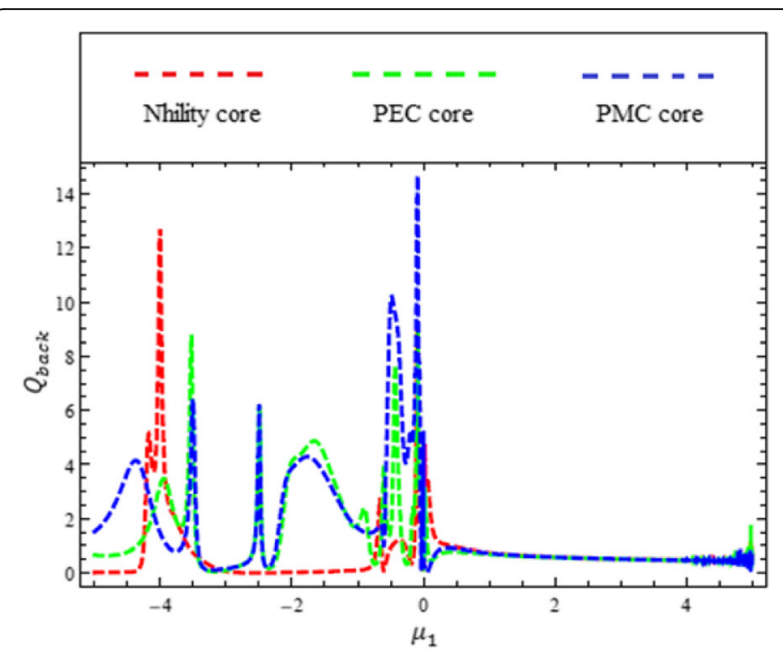

Fig. 14 Comparison between Back-Scattering efficiencies of nihility, PEC and PMC sphere as a function of $\mu_{1}$ when $\boldsymbol{\epsilon}_{1}=-2.54, a=0.4 \times \lambda$, $b=1.5 \times a, f=1 \mathrm{GHz}$

and PMC sphere only when the value of $\mu_{1}$ ranging between -5 to -4 , while show lower magnitude value as compared to PEC and PMC when $\mu_{1}=-4$ to 0 . The Back-Scattering efficiency of nihility sphere recorded maximum when $\mu_{1}=-4$, and minimum as compared to PEC and PMC at other values of $\mu_{1}$, which can be verified from Fig. 14. From these Figs, it was deduced that Forward-scattering and Extinction efficiencies of nihility depict higher magnitude than PEC and PMC in MNG coating case (at some specific values of permeability) and lower in ENG coating case as shown in Figs. 13, 14 and 15.

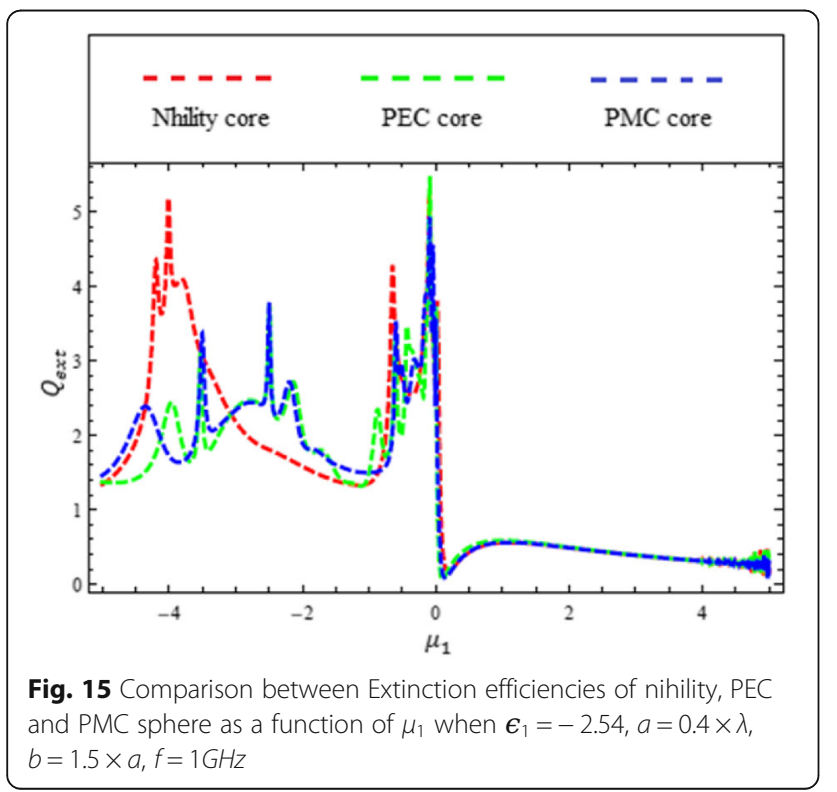

\section{Conclusions}

The problem of electromagnetic wave scattering from metamaterial coated nihility sphere by using extended classical wave theory have been mathematically formulated and analyzed. It is observed that the ForwardScattering, Back-scattering and Extinction efficiencies strongly depend on coating thickness. When we consider the DPS coating, these efficiencies depict higher magnitude than PEC and PMC sphere at higher values of coating thickness, while in DNG case scattering efficiencies following increasing pattern at lower values of coating thickness. It is furthermore reported that in DNG coating case, we observed that lower scattering efficiencies magnitude of Nihility sphere as compared to PEC and PMC at higher values of coating thickness. Based on the obtained results, we predicted that, scattering efficiencies can be controlled and tuned more effectively by producing metamaterial coating layer. These results may be very helpful in the defense technologies and optical illusion devices.

\section{Abbreviations \\ DNG: Double Negative; DPS: Double Positive; EMG: Epsilon Negative; MNG: Mu negative; PEC: Perfect Electric Conductor; PMC: Perfect Magnetic Conductor; SRR: Split Ring Resonator}

\section{Funding}

Deanship of Scientific Research (DSR) at King Saud University for its funding of this research through the Research Group no RG-1436-001.

\section{Availability of data and materials}

Detail about data has been provided in the article.

\section{Authors' contributions}

All authors contributed equally in all the sections of this work. All authors read and approved the final manuscript.

\section{Competing interests}

The authors declare that they have no competing interests.

\section{Author details}

${ }^{1}$ Department of Physics, University of Agriculture, Faisalabad, Pakistan.

${ }^{2}$ Department of Electrical Engineering, King Saud University, Riyadh, Saudi Arabia.

Received: 3 December 2016 Accepted: 15 February 2017

Published online: 17 March 2017

\section{References}

1. Capolino, F: Applications of metamaterials. CRC press, USA (2009)

2. Engheta, $\mathrm{N}$, and Ziolkowski, RW: Metamaterials: physics and engineering explorations. John Wiley \& Sons, USA (2006)

3. Gil, M., Bonache, J., Martin, F.: Metamaterial filters: A review. Metamaterials 2(4), 186-197 (2008)

4. Ding, F, and Yang, H: EM scattering by objects coated with DNM. In: Microwave, Antenna, Propagation and EMC Technologies for Wireless Communications (MAPE), 2013 IEEE 5th International Symposium on. IEEE, USA (2013)

5. Geng, Y-L: Mie Scattering by a Conducting Sphere Coated Uniaxial SingleNegative Medium. Int J Antennas Propagation. 2012(856476), 1-6 (2012)

6. Zhang, S., et al.: Negative refractive index in chiral metamaterials. Phys Rev Lett 102(2), 023901 (2009)

7. Afzaal, M., et al.: Scattering of electromagnetic plane wave by an impedance strip embedded in homogeneous isotropic chiral medium. Opt Commun $342,115-124$ (2015) 
8. Ghaffar, A., Alkanhal, M.A.: Electromagnetic waves in parallel plate uniaxial anisotropic chiral waveguides. Opt Mater Express 4(9), 1756-1761 (2014)

9. Sihvola, A.: Metamaterials in electromagnetics. Metamaterials 1(1), 2-11 (2007)

10. Lakhtakia, A: An electromagnetic trinity from "negative permittivity" and "negative permeability". arXiv preprint physics/0112003. Int J Infrared Millimeter Waves 22(12), 1731-1734 (2001)

11. Lakhtakia, A., Geddes lii, J.B.: Scattering by a nihility cylinder. AEU-Int J Electron Commun 61(1), 62-65 (2007)

12. Lakhtakia, A.: Scattering by a nihility sphere. Microwave Opt Technol Lett 48(5), 895-896 (2006)

13. Lakhtakia, A.: Radiation pressure efficiencies of spheres made of isotropic, achiral, passive, homogeneous, negative-phase-velocity materials. Electromagnetics 28(5), 346-353 (2008)

14. Capretti, A., et al.: Comparative study of second-harmonic generation from epsilon-near-zero indium tin oxide and titanium nitride nanolayers excited in the near-infrared spectral range. ACS Photonics 2(11), 1584-1591 (2015)

15. Liberal, I., Engheta, N.: Nonradiating and radiating modes excited by quantum emitters in open epsilon-near-zero cavities. Sci Adv 2(10), e1600987 (2016)

16. Liberal, I, and Engheta, $\mathrm{N}$ : Selected features of metamaterials with near-zero parameters. In: Electromagnetic Theory (EMTS), 2016 URSI International Symposium on. IEEE, USA (2016)

17. Liberal, I., Engheta, N.: Zero-Index Platforms: where light defies geometry. Opt Photonic News 27(7), 26-33 (2016)

18. Dong, J.F., Li, J.: Characteristics of guided modes in uniaxial chiral circular waveguides. Prog Electromagnetics Res 124, 331-345 (2012)

19. Ahmed, S., Naqvi, Q.A.: Scattering of electromagnetic waves by a coated nihility cylinder. J Infrared Millimeter Terahertz Waves 30(10), 1044-1052 (2009)

20. Ahmed, S., Naqvi, Q.A.: Electromagnetic scattering from a chiral-coated nihility cylinder. Prog Electromagnetics Res Lett 18, 41-50 (2010)

21. Shoukat, S., et al.: Scattering from a coated nihility circular cylinder placed in chiral metamaterial. Optik-Int J Light Electron Opt 125(15), 3886-3890 (2014)

22. Yaqoob, M., et al.: Scattering of electromagnetic waves from a chiral coated nihility cylinder hosted by isotropic plasma medium. Opt Mater Express 5(5), 1224-1229 (2015)

23. Bohren, CF, and Huffman, DR: Absorption and Scattering of Light by Small Particle, John Wiley \& Sons, USA (1983)

24. Stratton, J: Electromagnetic Theory, McGrow-Hill, New-York. London (1941)

\section{Submit your manuscript to a SpringerOpen ${ }^{\circ}$ journal and benefit from:}

- Convenient online submission

- Rigorous peer review

- Immediate publication on acceptance

- Open access: articles freely available online

- High visibility within the field

Retaining the copyright to your article

Submit your next manuscript at $\gg$ springeropen.com 